\title{
Diagnostic performance and clinical application of preoperative COVID-19 bedside testing with ID NOW ${ }^{\mathrm{TM}}$
}

\author{
Tonia Tauh, MD, FRCPC · Susan M. Lee, MD, MAS, FRCPC (iD · Paula Meyler, MD, \\ FRCPC • Michelle Mozel, MSc $\cdot$ Meghan McLennan, BSc, MLT $\cdot$ Linda M. N. Hoang, MD, \\ MSc, DTM\&H, FRCPC
}

Received: 4 May 2021/Revised: 6 May 2021/Accepted: 6 May 2021/Published online: 21 May 2021

(C) Canadian Anesthesiologists' Society 2021

To the Editor,

We would like to report findings on the diagnostic performance of the Abbott ID NOW ${ }^{\mathrm{TM}}$ COVID-19 pointof-care molecular test (Abbott Diagnostics Scarborough, Inc., Scarborough, ME, USA) when used in largely asymptomatic urgent preoperative patients. Laboratory nucleic acid testing (NAT) of nasopharyngeal (NP) swabs is considered the gold standard to assess for the presence of SARS-CoV-2 RNA. Whereas laboratory results can take 24-72 hr, the ID NOW COVID-19 test provides results in circa $15 \mathrm{~min}$. The diagnostic accuracy of the ID NOW COVID-19 assay has been assessed in a recent Cochrane review, which reported a sensitivity of $73.0 \%$ and specificity of $99.7 \%$; the latter was slightly higher than that of the Cepheid Xpert ${ }^{\circledR}$ Xpress SARS-CoV-2 test (Cepheid, Sunnyvale, CA, USA) while the former was lower. ${ }^{1}$ Nevertheless, most of the tests were conducted in symptomatic or high-risk patients, and test performance in asymptomatic populations has not been widely reported.

T. Tauh, MD, FRCPC - S. M. Lee, MD, MAS, FRCPC ( $₫)$.

P. Meyler, MD, FRCPC $\cdot$ M. Mozel, MSc

Department of Anesthesia and Perioperative Medicine, Royal

Columbian Hospital, New Westminster, BC, Canada

e-mail: suze.lee@utoronto.ca

Department of Anesthesiology, Pharmacology \&Therapeutics, The University of British Columbia, Vancouver, BC, Canada

M. McLennan, BSc, MLTL. M. N. Hoang, MD, MSc, DTM\&H, FRCPC

BC Centre for Disease Control Public Health Laboratory,

Vancouver, BC, Canada

Department of Pathology \& Laboratory Medicine, The

University of British Columbia, Vancouver, BC, Canada
The Royal Columbian Hospital ( $\mathrm{RCH}$ ) operating room was the first in British Columbia to pilot the ID NOW COVID-19 assay for bedside preoperative COVID-19 testing. The clinical validation project was exempt from research ethics board approval. During this time, British Columbia was at the tail of its second wave, averaging a weekly incidence of 78 per 100,000. The Fraser Health region, where $\mathrm{RCH}$ is located, was the most affected region, with a weekly incidence of 117 per 100,000 and test positivity rates around $10.1 \% .^{2}$

From December 2020 to April 2021, ID NOW COVID19 tests were administered to 1,100 urgent surgical patients who were largely asymptomatic. Concurrent NP swabs were also sent for a comparative standard NAT. There were 1,093 true negative tests, two true positives, two false negatives, and three false positives (Table). The test had a sensitivity of $50 \%$ (95\% confidence interval [CI], 6.8 to 93.2), specificity of $99.7 \%$ (95\% CI, 99.2 to 99.4 ), positive predictive value of $40.0 \%$ (95\% CI, 13.0 to 74.8 ), and negative predictive value of $99.8 \%$ (95\% CI, 99.5 to 99.9 ); the prevalence was $0.36 \%$ (95\% CI, 0.10 to 0.93 ).

An immediate repeat of the ID NOW COVID-19 test was performed on all ID NOW COVID-19-positive results. The three false positives yielded negative repeat results while both true positives remained positive when repeated. When considering only repeated positive ID NOW COVID-19 test results as true positives, the specificity increased to $100 \%$ (95\% CI, 99.7 to 100 ).

Low cycle threshold $(\mathrm{Ct})$ values reflect higher viral loads and likely increased infectivity. ${ }^{3}$ Both false negatives had high Ct values (29.5 and 30.0, respectively [E gene]). Discordant results at higher $\mathrm{Ct}$ values $(>25)$ are consistent with previous reports. ${ }^{1}$ While the NAT is a more sensitive test, if the goal is to identify only the most highly infectious (i.e., those with a low Ct value), the ID NOW COVID-19 
TABLE Performance summary of the ID NOW COVID-19 test

\begin{tabular}{lll}
\hline A. & & NAT/NP \\
\cline { 2 - 3 } & & Positive \\
\hline ID NOW COVID-19 & Positive & 2 \\
& Negative & 2 \\
Sensitivity & $50 \%(95 \% \mathrm{CI}, 6.8$ to 93.2$)$ & \\
Specificity & $99.7 \%(95 \% \mathrm{CI}, 99.2$ to 99.9$)$ & \\
Positive likelihood ratio & $182.7(95 \% \mathrm{CI}, 40.9$ to 815.2$)$ & \\
Negative likelihood ratio & $0.50(95 \% \mathrm{CI}, 0.19$ to 1.34$)$ & \\
Positive predictive value & $40.0(95 \% \mathrm{CI}, 13.0$ to 74.8$)$ & \\
Negative predictive value & $99.8(95 \% \mathrm{CI}, 99.5$ to 99.9$)$ & \\
\hline
\end{tabular}

B.

NAT/NP

Positive Negative (considered negative if $\mathbf{C t} \mathbf{~} \mathbf{2 5}$ )

ID NOW COVID-19 performance with modified definitions* Positive (on repeat sample) 2 0 Negative (not repeated) 0 1,098

Sensitivity

Specificity

Positive predictive value

Negative predictive value
$100 \%$ (95\% CI, 15.8 to 100$)$

$100 \%(95 \% \mathrm{CI}, 99.7$ to 100$)$

$100 \%(34.2-100 \%)$

$100 \%(99.7-100 \%)$

* ID NOW COVID-19 considered positive only after two positive results on separate samples and laboratory NAT considered negative if Ct $>25$ $\mathrm{CI}=$ confidence interval; $\mathrm{Ct}=$ cycle threshold; NAT/NP = nucleic acid testing/nasopharyngeal

A) Samples with single ID NOW COVID-19 test; B) Samples with positives repeated by ID NOW COVID-19

performs well: considering $\mathrm{Ct}$ values $<25$ only to be positive, the sensitivity is improved to $100 \%$ (95\% CI, 15.8 to 100$)$. One patient was excluded from the analysis who tested positive one month preoperatively, negative (NAT) $48 \mathrm{hr}$ preoperatively, and had a presumed residual positive NAT on the day of surgery (E gene $\mathrm{Ct}$ value, 32.7 ) at the time of a negative ID NOW test, which, by protocol, should not have been performed.

Both true positives had E gene Ct values $<25$ (15.7 and 18.1, respectively). Notably, the second patient had tested negative (NAT) $48 \mathrm{hr}$ prior at another hospital, indicating a possible nosocomial infection or a viral load below the limit of detection $48 \mathrm{hr}$ prior. Both patients that were found positive by the ID NOW were asymptomatic preoperatively and both developed symptoms postoperatively while convalescing on the COVID-19 ward.

When the COVID-19 status of a patient is unknown, confusion and inconsistent use of resources may occur. Ideally, a patient's infectious status should be known so that optimal personal protective equipment (PPE), aerosol minimizing techniques, and patient flow through the hospital can be adopted. In the clinical setting, patients that test negative on the rapid test in conjunction with a negative screening questionnaire often receive standard precautions, despite a chance that the negative test result could be incorrect. This could result in a false sense of security with relaxed infectious precautions. Nevertheless, in the setting of using ID NOW COVID-19 in a lowprevalence population, the risk of transmission due to false negatives is likely low; the high negative predictive value of the test coupled with a false negative's likely noninfectious nature, as reflected by a high $\mathrm{Ct}$ value, should provide ample reassurance. Conversely, false positives can lead to increased resource consumption through higher PPE utilization, misallocation of isolation rooms, as well as delays in non-emergent treatments and differential nursing bedside care. However, false positives were minimized with repeated ID NOW COVID-19 testing of all positives results.

The ID NOW COVID-19 test has limited sensitivity; however, since it accurately identifies patients with more infectious low $\mathrm{Ct}$ values that correspond to a higher viral load, this may be acceptable. Its specificity is acceptable, particularly when requiring repeat positive tests to be considered true positives.

Overall, the ID NOW COVID-19 performed well in a primarily asymptomatic preoperative population, and likely performs better with a higher population prevalence. In situations where urgent invasive intervention is 
required before NP results can be established and a positive result would change precautions management and workflow, the ID NOW COVID-19 test should be considered to assist with early identification of potentially infectious COVID-19 patients.

Acknowledgements Thank you to the British Columbia Centre for Disease Control Public Health Laboratory. Many thanks also to the operating room staff at Royal Columbian Hospital for trialing the IDNow in addition to their tireless work throughout the pandemic.

Disclosures None.

Funding statement This work was supported by the Royal Columbian Hospital Foundation Anesthesia Innovation Fund. IDNow tests were provided by the British Columbia Centre for Disease Control.

Editorial responsibility This submission was handled by Dr. Philip M. Jones, Deputy Editor-in-Chief, Canadian Journal of Anesthesia/ Journal canadien d'anesthésie.

\section{References}

1. Dinnes J, Deeks JJ, Berhane $S$, et al. Rapid, point-of-care antigen and molecular-based tests for diagnosis of SARS-CoV-2 infection. Cochrane Database Syst Rev 2021. Doi: https://doi.org/10.1002/ 14651858.CD013705.pub2.

2. BC Center for Disease Control. British Columbia (BC) COVID-19 Situation Report Week 11 (Mar 14 - Mar 20, 2021). Available from URL: http://www.bccdc.ca/Health-Info-Site/Documents/ COVID_sitrep/Week_11_2021_BC_COVID-19_Situation_ Report_Final.pdf (accessed May 2021).

3. Singanayagam A, Patel $M$, Charlett A, et al. Duration of infectiousness and correlation with RT-PCR cycle threshold values in cases of COVID-19, England, January to May 2020. Euro Surveill 2020. Doi: https://doi.org/10.2807/1560-7917.ES. 2020.25.32.2001483.

Publisher's Note Springer Nature remains neutral with regard to jurisdictional claims in published maps and institutional affiliations. 